\title{
CLASSIFICATION OF CERTAIN QUALITATIVE PROPERTIES OF SOLUTIONS FOR THE QUASILINEAR PARABOLIC EQUATIONS
}

\author{
YAN LI, ZHENGCE ZHANG AND LIPING ZHU
}

\begin{abstract}
In this paper, we mainly consider the initial boundary problem for a quasilinear parabolic equation

$$
u_{t}-\operatorname{div}\left(|\nabla u|^{p-2} \nabla u\right)=-|u|^{\beta-1} u+\alpha|u|^{q-2} u,
$$

where $p>1, \beta>0, q \geq 1$ and $\alpha>0$. By using Gagliardo-Nirenberg type inequality, energy method and comparison principle, the phenomena of blowup and extinction are classified completely in the different ranges of reaction exponents.
\end{abstract}

\section{INTRODUCTION}

In this paper, the following initial boundary problem is considered:

$$
\left\{\begin{array}{rlrl}
u_{t}-\Delta_{p} u & =-|u|^{\beta-1} u+\alpha|u|^{q-2} u, & x \in \Omega, t & >0, \\
u & =0, & x \in \partial \Omega, t & >0, \\
u(x, 0) & =u_{0}(x), & x \in \Omega,
\end{array}\right.
$$

where $\Omega \subset \mathbb{R}^{N}(N \geq 1)$ is a smoothly bounded domain and $p>1, \beta>0, q \geq 1, \alpha>0$. The operator $\Delta_{p}$ is defined as follows:

$$
\Delta_{p} u:=\operatorname{div}\left(|\nabla u|^{p-2} \nabla u\right) .
$$

We also suppose that $u_{0}(x) \geq 0, u_{0}(x) \not \equiv 0, u_{0}(x) \in W_{0}^{1, p}(\Omega) \cap L^{\infty}(\Omega)$.

Problem (1.1) arises in the theory of nonstationary filtration of non-Newtonian (or dilatant) fluids and combustion of solid fuels. The term $-|u|^{\beta-1} u$, which is negative as we can prove later that $u \geq 0$, is called a singular absorption term for $\beta<0$ or a strong absorption one for $0<\beta<1$ or a weak absorption one for $\beta>1 . \alpha|u|^{q-2} u$ is an inner source term. It has been known for many years that the term $-|u|^{\beta-1} u$ with $\beta>0$ may lead to finite time extinction, i.e. there exists a $T \in(0,+\infty)$ such that $u(x, t)$ is nontrivial for $t \in[0, T)$ and $u(x, t) \equiv 0$ for $t \in[T,+\infty)$ a.e. in $x \in \Omega$. On the other hand, $f(x, u)$ may lead to finite time blowup. However, if the two terms appear simultaneously in the first equation of (1.1), then the solutions will exhibit complicated properties which will be studied later. To be specific, both blowup and extinction can occur under some suitable conditions.

Date: September 25, 2018.

Corresponding author: Zhengce Zhang.

Keywords: Qusilinear parabolic equation, Weak solution, Blowup, Extinction.

2010 Mathematics Subject Classification: 35A01, 35B44, 35D30, 35K92. 
As the operator $\Delta_{p}$ is degenerate for $p>2$ and is singular for $1<p<2$, it's impossible to consider the classical solution of (1.1) generally. However, the concept of weak solution is enough for our study. For the local existence of weak solution of (1.1), there are various methods can be applied such as approximation by regular solution [4, 36], fixed point method [33], the method of extension of semigroup [12] and the developed Faedo-Galerkin method [2, 3, 14.

As soon as the local existence is established, one may ask whether the weak solution is global or not. Moreover, we are eager to know when the solution is global in time and when it blows up in finite time. For the global solution, we also want to know whether it will become zero in finite time or not.

The phenomenon of finite time blowup was first considered by H. Fujita [1] in 1966. Since then, many people devoted themselves to this problem. The main equation they studied is the heat equation of the form $u_{t}-\Delta u=|u|^{p-1} u$ in bounded or unbounded smooth domain in $\mathbb{R}^{N}$. The theory of blowup for heat equation is already developed, we refer the reader to [16, 19, 25, 26, 28] and the references therein. While for the $p$-Laplacian equations of the form $u_{t}-\Delta_{p} u=f(x, t, u, \nabla u)$, there are still many problems worth studying, such as the blowup rate, the blowup time estimate, the asymptotic behavior of blowup solutions, the blowup criteria and so on. Some related results can be found in [13, 14, 20, 24, 33, 34, 35, 36, 39, 37] and the references therein. To be specific, in [14, 20, 24, 33, 36], criteria for the finite time blow-up to occur were established in bounded domain for different kinds of source terms and values of $p$. Generally speaking, finite time blowup may occur if $f(x, t, s, \vec{r})$ grows faster than $s^{p-1}(p>2)$ or $s(1<p<2)(q=p-1$ or $q=1$ is called the critical blowup exponent) when $s \rightarrow \infty$ and the initial data is large enough. In [13], Galaktionov and Posashkov studied the blowup set for the equation $u_{t}-\operatorname{div}\left(|\nabla u|^{\sigma} \nabla u\right)=u^{\beta}$ with $\sigma>0, \beta>1$ and $x \in \mathbb{R}^{N}$. They proved that the radial solution will blow up at $|x|=0$. For the blowup time estimate, Zhou and Yang [39] considered the equation $u_{t}-\operatorname{div}\left(|\nabla u|^{m-2} \nabla u\right)=|u|^{p(x)-1} u$ with Dirichlet boundary condition on bounded domains. They obtained a upper bound of the blowup time for some suitable conditions on $m, p(x)$ and initial data. and Zhao and Liang [37] considered a Cauchy problem $u_{t}-\Delta_{p} u=u^{q}$ in the radial situation and obtained the blowup rate upper bound is of the order $(T-t)^{-1 /(q-1)}$ for $q>p-1$. In our latest papers [34, 35], we considered the equation $u_{t}-\Delta_{p} u=\lambda u^{m}+\mu|\nabla u|^{q}$ with $p>2$ and $\lambda \mu<0$, and proved that $u$ will blow up in finite time in the $L^{\infty}$-norm sense if $\lambda>0, \mu<0$ and $m>\max \{p-1, q\}, q \leq p / 2$. For the blowup of more general p-Laplacian equations, there are also some important results. In [30, 32, the Fujita exponent for equations with weighted source of the form

$$
\frac{\partial u}{\partial t}=\operatorname{div}\left(|\nabla u|^{p-2} \nabla u\right)+k \frac{1}{|x|^{2}}|\nabla u|^{m-1} \nabla u \cdot x+|x|^{\lambda} u^{p}
$$

were studied. In [23, 38], the global existence, blowup and the blowup point of solutions for the doubly degenerate equations, i.e. equations with $\operatorname{div}\left(\left|\nabla u^{m}\right|^{p-2} \nabla u^{m}\right)$ were carefully studied. 
Finite time extinction is another important property of solutions of evolution equations. Since Kalashnikov first brought in the concept of extinction in 1974, it has attracted many mathematicians' interests and most of them focused on the fast diffusive equations, see [6, 7, 8, 9, 10, 15, 18, 31, 33] for examples. Moreover, in [29], the homogeneous $p$-Laplacian equation $u_{t}=\Delta_{p} u$ with $p>1, x \in \mathbb{R}^{N}$ was studied. It was shown that extinction can happen if and only if $1<p \leq p_{c}=2 N /(N+1)$. In [33], Yin and Jin considered the equation $u_{t}-\Delta_{p} u=\lambda u^{q}$ with $x \in \Omega$ and $1<p<2$. They proved that $q=p-1$ is the critical extinction exponent. In [15], Gu considered the $p$-Laplacian equation $u_{t}-\Delta_{p} u=-|u|^{\beta-1} u$ with $p>1$. In that paper, the conditions for extinction to occur were obtained for any $p>1$ while the non-extinction condition was obtained only for $p \geq 2$. For the equation with absorption and source terms, i.e. $u_{t}-\Delta_{p} u=\lambda u^{q}-\beta u^{k}$ with $1<p<2$ and $0<q, k<1$, it was showed in [8] that the solution will exhibit extinction phenomenon under the assumptions that $u_{0}(x)$ or $\lambda$ is small enough and that $\beta$ is large enough. In [17, 24, the extinction phenomenon for p-Laplacian with Neumann boundary data and nonlocal absorption term were studied.

In this paper, we will deal with problem (1.1) for any $p>1$. In Section 2, we will give some basic concepts and a weak comparison principle. Section 3 is devoted to the existence of the weak solution for problem (1.1) in a general case. The extinction phenomenon will be discussed in Section 4. At last, we will give some blowup results under different conditions for $u_{0}(x)$ and $p, \beta, q, \alpha$.

\section{Preliminaries}

Before giving the definition of weak solution, we bring in the following function space:

$$
\mathbb{V}:=\left\{v \in L^{p}\left(0, T ; W_{0}^{1, p}(\Omega)\right) \mid \partial_{t} v \in L^{p^{\prime}}\left(0, T ; W^{-1, p^{\prime}}(\Omega)\right)\right\} .
$$

Now, let us introduce the definition of weak solution of (1.1).

Definition 2.1. Let $Q_{T}=\Omega \times(0, T), S_{T}=\partial \Omega \times(0, T), \partial Q_{T}=S_{T} \cup\{\bar{\Omega} \times\{0\}\}$. A function $u \in \mathbb{V} \cap C\left(0, T ; L^{2}(\Omega)\right)$ is called a weak solution of (1.1) if it satisfies:

1. for every nonnegative test-function $\varphi \in \mathbb{V} \cap C\left(0, T ; L^{2}(\Omega)\right)$,

$$
\iint_{Q_{T}}\left(\partial_{t} u \varphi+|\nabla u|^{p-2} \nabla u \cdot \nabla \varphi\right) \mathrm{d} x \mathrm{~d} t=-\iint_{Q_{T}}\left(|u|^{\beta-1} u-\alpha|u|^{q-2} u\right) \varphi \mathrm{d} x \mathrm{~d} t .
$$

2. $u(x, 0)=u_{0}(x)$ for a.e. $x \in \Omega$.

Moreover, if we replace "=" in (2.2) by " $\leq "$ " " $\geq ")$ and assume that $u(x, 0) \leq(\geq$ )$u_{0}(x),\left.u(x, t)\right|_{x \in \partial \Omega} \leq(\geq) 0$, then the corresponding solution is called a sub-(sup-) solution.

For the weak solution of (1.1), we have the following weak comparison principle. Some similar results can be found in [4, 20, 33, 34].

Proposition 2.1. Suppose that $u, v$ are weak sub-and sup-solutions of (1.1) respectively. If $u$ and $v$ are locally bounded, then $u \leq v$ a.e. in $Q_{T}$. 
Proof. Let $\varphi=\max \{u-v, 0\}$, then $\varphi(x, 0)=0,\left.\varphi(x, t)\right|_{x \in \partial \Omega}=0$. By Definition 2.1, $\varphi(x, t)$ satisfies:

$$
\begin{aligned}
& \iint_{Q_{T}} \partial_{t} \varphi \varphi \mathrm{d} x \mathrm{~d} t+\underbrace{\iint_{Q_{T}}\left(|\nabla u|^{p-2} \nabla u-|\nabla v|^{p-2} \nabla v\right)(\nabla u-\nabla v) \mathrm{d} x \mathrm{~d} t}_{\mathscr{M}} \\
\leq & -\iint_{Q_{T}}\left(|u|^{\beta-1} u-|v|^{\beta-1} v\right) \varphi \mathrm{d} x \mathrm{~d} t+\alpha \iint_{Q_{T}}\left(|u|^{q-2} u-|v|^{q-2} v\right) \varphi \mathrm{d} x \mathrm{~d} t \\
\leq & -\underbrace{\iint_{Q_{T}}\left(|u|^{\beta-1} u-|v|^{\beta-1} v\right) \varphi \mathrm{d} x \mathrm{~d} t}_{\mathscr{A}}+L \iint_{Q_{T}} \varphi^{2} \mathrm{~d} x \mathrm{~d} t
\end{aligned}
$$

where $L$ is a constant depending on the sup-norms of $u$ and $v$.

Let us now estimate terms $\mathscr{M}$ and $\mathscr{A}$ appearing in (2.3). By the monotone inequality (see [21]), we have $\mathscr{M} \geq 0$ for any $p>1$. For term $\mathscr{A}$, by the fact that

$$
\left\{\begin{array}{l}
|u|^{\beta-1} u-|v|^{\beta-1} v=u^{\beta}-v^{\beta}>0, \text { if } u>v>0, \\
|u|^{\beta-1} u-|v|^{\beta-1} v=u^{\beta}+|v|^{\beta}>0, \text { if } u>0>v, \\
|u|^{\beta-1} u-|v|^{\beta-1} v=-|u|^{\beta}+|v|^{\beta}>0, \text { if } 0>u>v,
\end{array}\right.
$$

we have $\mathscr{A} \geq 0$.

Following the discussion above, we have

$$
\frac{1}{2} \int_{\Omega} \varphi^{2} \mathrm{~d} x \leq L \iint_{Q_{T}} \varphi^{2} \mathrm{~d} x \mathrm{~d} t .
$$

By Gronwall's inequality, we have $\int_{\Omega} \varphi^{2} \mathrm{~d} x=0$. This implies that $\varphi=0$ a.e. $x \in \Omega$, i.e. $u \leq v$ a.e. $(x, t) \in Q_{T}$.

\section{Existence OF WEAK SOLUTiOn}

In this section, we will establish the local existence and global existence of weak solutions of (1.1). Analogous to the proofs in [2, 3, 14] and the compactness results in [27], we have the following local existence of bounded weak solution for (1.1).

Theorem 3.1. Suppose that $u_{0} \in W_{0}^{1, p}(\Omega) \cap L^{\infty}(\Omega), u_{0} \geq 0, u_{0} \not \equiv 0$ a.e. in $\Omega$ and that $q \geq 1$. Then there exists a $T^{*}=T^{*}\left(u_{0}\right)>0$ such that for $0<T<T^{*}$ (1.1) admits a solution

$$
u \in \mathbb{U}:=\left\{u \in L^{\infty}\left(0, T ; W_{0}^{1, p}(\Omega)\right) \cap L^{\infty}\left(Q_{T}\right) \mid \partial_{t} u \in L^{2}\left(Q_{T}\right)\right\} .
$$

Moreover, $0 \leq u \leq M$ a.e. in $Q_{T}$ for some $M$ depending on $u_{0}(x)$.

Next, we will give some results focusing on the global existence of the weak solution for (1.1). 
Denote by $\Lambda_{1}>0$ the first eigenvalue of the $p$-Laplacian operator with homogeneous Dirichlet boundary condition, i.e.

$$
\Lambda_{1}:=\inf \left\{\left.\int_{\Omega}|\nabla u|^{p} \mathrm{~d} x\left|u \in W_{0}^{1, p}(\Omega), \int_{\Omega}\right| u\right|^{p} \mathrm{~d} x=1\right\} .
$$

Theorem 3.2 (Global existence). Let $u_{0}(x) \in W_{0}^{1, p}(\Omega) \cap L^{\infty}(\Omega), u_{0}(x) \geq 0$ and one of the following conditions is satisfied

(i) $q=p, \alpha<\Lambda_{1}$.

(ii) $q=p=\beta+1, \alpha<\Lambda_{1}+1$.

(iii) $2<p \leq q<\beta+1$.

(iv) $q<p$.

Then the solution of (1.1) is globally in time bounded, i.e. there exists a constant $M$ depends only on $p, q, \beta, \Lambda_{1}, \alpha, u_{0}, \Omega$ such that for every $T>0,0 \leq u \leq M$.

Proof. Case (i). Let $\widetilde{\Omega} \subset \mathbb{R}^{N}$ be a smooth domain which satisfies: $\Omega \subset \subset \widetilde{\Omega}$. Denote by $\phi$ and $\Lambda_{1}(\widetilde{\Omega})$ the first eigenfunction and the first eigenvalue related to the following Dirichlet problem:

$$
-\Delta_{p} \phi=\Lambda_{1}(\widetilde{\Omega})|\phi|^{p-2} \phi \text { in } \widetilde{\Omega}, \phi=0 \text { on } \partial \widetilde{\Omega}, \int_{\widetilde{\Omega}}|\phi|^{p} \mathrm{~d} x=1 .
$$

Then by [20, Lemma 1.1], we know that $\phi>0$ in $\widetilde{\Omega}$ and that $\Lambda_{1}(\widetilde{\Omega})<\Lambda_{1}(\Omega)$. Moreover, by [22, Theorem 3.2], $\Lambda_{1}(\widetilde{\Omega})$ continuously depends on $\widetilde{\Omega}$ and $\Lambda_{1}(\widetilde{\Omega}) \rightarrow$ $\Lambda_{1}(\Omega)$ as $\widetilde{\Omega} \rightarrow \Omega$ in the Hausdorff complementary topology. Thus, we can choose a suitable $\widetilde{\Omega}$ and $\theta>0$ such that $\alpha \leq \Lambda_{1}(\widetilde{\Omega}) \leq \Lambda_{1}(\Omega)$. Let $\Phi=K \phi \geq K \mu \geq\left\|u_{0}\right\|_{L^{\infty}(\Omega)}$ with $\mu=\inf _{\Omega} \phi>0$. Then a simple calculation shows that for every nonnegative test-function $\varphi \in \mathbb{V} \cap\left(0, T ; L^{2}(\Omega)\right)$

$$
\begin{aligned}
\iint_{Q_{T}} \partial_{t} \Phi \varphi+|\nabla \Phi|^{p-2} \nabla \Phi \cdot \nabla \varphi \mathrm{d} x \mathrm{~d} t & \geq \Lambda_{1}(\widetilde{\Omega}) \iint_{Q_{T}} \Phi^{p-1} \varphi \mathrm{d} x \mathrm{~d} t \\
& \geq \alpha \iint_{Q_{T}} \Phi^{p-1} \varphi \mathrm{d} x \mathrm{~d} t
\end{aligned}
$$

This implies that $\Phi$ is a sup-solution of (1.1). Then by Proposition 2.1, we have $0 \leq u \leq \Phi$ a.e. in $Q_{T}$. We can also see from the construction of $\Phi$ that it's independent of $t$ which enables us to continue the procedure above on any time interval $\left[T, T^{\prime}\right]$. Then, we can assert that the solution of (1.1) is globally in time bounded.

The proof of Case (ii) is same as the one of Case (i).

Case (iii). Without loss of generality, we assume $\alpha=1$, the method below is still valid for the general case with a little modification. Denote by $\rho(\Omega)$ the diameter of $\Omega$, then we can easily know that $\rho(\Omega)<\infty$ as $\Omega$ is bounded. Let $\varepsilon \in(0,1)$ satisfies: there exists a ball of radius $\varepsilon$ belonging to $B(\cdot, \rho(\Omega)+1) \cap \Omega^{c}$. For any $a \in \Omega$, let $x_{a}$ satisfies:

$$
B\left(x_{a}, \varepsilon\right) \subset B\left(x_{a}, \rho(\Omega)+1\right) \cap \Omega^{c},\left|x_{a}-a\right|<\rho(\Omega)+1 .
$$


Let

$$
V(x, t)=L \mathrm{e}^{\sigma r}, r=\left|x-x_{a}\right|, x \in \Omega .
$$

Define: $\mathscr{L}_{p} v:=v_{t}-\Delta_{p} v-v^{q-1}+v^{\beta}$, then $V(x, t)$ satisfies

$$
\mathscr{L}_{p} V=-(p-1)(L \sigma)^{p-1} \mathrm{e}^{(p-1) \sigma r}-\frac{N-1}{r}(L \sigma)^{p-1} \mathrm{e}^{(p-1) \sigma r}-L^{q-1} \mathrm{e}^{(q-1) \sigma r}+L^{\beta} \mathrm{e}^{\beta \sigma r} .
$$

In order to derive that $\mathscr{L}_{p} V \geq 0$, we need to choose suitable $\sigma$ and $L$ such that

$$
(p-1) \sigma^{p}+\frac{N-1}{r} \sigma^{p-1} \leq L^{\beta+1-p} \mathrm{e}^{(\beta+1-p) \sigma r}-L^{q-p} \mathrm{e}^{(q-p) \sigma r} .
$$

By (3.5) and (3.6), we know that $\varepsilon \leq r<\rho(\Omega)+1$. Then if we want (3.8) to be satisfied, it's sufficient that

$$
(p-1) \sigma^{p}+\frac{N-1}{\varepsilon} \sigma^{p-1}+L^{q-p} \mathrm{e}^{(q-p) \sigma(\rho(\Omega)+1)} \leq L^{\beta+1-p} .
$$

If $q>p$, let $\sigma$ and $L$ satisfy

$$
\sigma=\frac{1}{(q-p)(\rho(\Omega)+1)}, L=\max \left\{(2 \mathrm{e})^{\frac{1}{\beta+1-q}},\left(2\left((p-1) \sigma^{p}+\frac{N-1}{\varepsilon} \sigma^{p-1}\right)\right)^{\frac{1}{\beta+1-p}}\right\} .
$$

While if $q=p$, let $\sigma$ and $L$ satisfy

$$
\sigma=1, L=\max \left\{2^{\frac{1}{\beta+1-q}},\left(2\left(p-1+\frac{N-1}{\varepsilon}\right)\right)^{\frac{1}{\beta+1-p}}\right\} .
$$

Then there holds $\mathscr{L}_{p} V \geq 0$. If we assume furthermore that $L \geq\left\|u_{0}\right\|_{L^{\infty}(\Omega)}$, then $V(x, 0) \geq u_{0}(x)$. Thus, we have proved that $V(x, t)$ is a super-solution of (1.1). By Proposition 2.1, we have

$$
u(x, t) \leq L \mathrm{e}^{\sigma(\rho(\Omega)+1)}<\infty .
$$

Notice that the right hand side of (3.12) is in fact independent of $t$, which enable us to continue the procedure above in any time interval $\left[T, T^{\prime}\right]$. Hence, we can conclude that $u(x, t)$ is globally in time bounded.

In the case $q<p$, by Young's inequality, there exists a small $\gamma>0$ such that $\alpha|s|^{q-2} s \leq \gamma|s|^{p-1}+C(\gamma)$. Then the conclusion follows from the same procedure as above.

\section{Finite time extinction and Decay}

Before proving our main results, we first introduce the following Gagliardo-Nirenberg type inequality which can be found in [7, 14] and the references therein.

Lemma 4.1. Let $1<p<+\infty$ and $r \in[\beta+1,+\infty)$ if $p \geq N$, and $r \in\left[\beta+1, \frac{N p}{N-p}\right]$ if $p<N$. Then there exists a constant $C>0$, depending only on $p, r, N, \beta$ and $|\Omega|$, 
such that for every $u \in W_{0}^{1, p}(\Omega)$

$$
\|u\|_{L^{r}(\Omega)} \leq C\|\nabla u\|_{L^{p}(\Omega)}^{\theta}\|u\|_{L^{\beta+1}(\Omega)}^{1-\theta} \text { with } \theta=\frac{\frac{1}{\beta+1}-\frac{1}{r}}{\frac{1}{N}-\frac{1}{p}+\frac{1}{\beta+1}} \in[0,1] .
$$

Remark 4.1. We can see from the expression of $\theta$ with $r>\beta+1$ that

$$
\theta<\frac{\frac{1}{\beta+1}-\frac{1}{r}}{-\frac{1}{p}+\frac{1}{\beta+1}}
$$

and that

$$
r\left(\frac{\theta}{p}+\frac{1-\theta}{\beta+1}\right)>1
$$

which will play an important role in establishing a desired ordinary differential inequality later.

4.1. Finite time extinction. The following theorem deals with the finite time extinction.

Theorem 4.1. Let $\beta+1 \leq q \leq p$ and $\beta<\min \{1, p-1\}$. Assume additionally that $\alpha<\min \left\{1, \Lambda_{1}\right\}$. Then there exists a finite time $T^{*}>0$, such that $u=0$ a.e. in $\Omega$ for $t \geq T^{*}$.

Proof. By Theorem [3.2, $u$ exists globally in time. Let $y(t)=\|u\|_{L^{2}(\Omega)}^{2}$. Then it satisfies:

$$
\frac{1}{2} y^{\prime}(t)+\int_{\Omega}|\nabla u|^{p} \mathrm{~d} x=\alpha \int_{\Omega} u^{q} \mathrm{~d} x-\int_{\Omega} u^{\beta+1} \mathrm{~d} x .
$$

By the assumption that $\beta+1 \leq q \leq p$, we have

$$
\begin{aligned}
\int_{\Omega} u^{q} \mathrm{~d} x & =\int_{\Omega \cap\{u \geq 1\}} u^{q} \mathrm{~d} x+\int_{\Omega \cap\{u \leq 1\}} u^{q} \mathrm{~d} x \leq \int_{\Omega \cap\{u \geq 1\}} u^{p} \mathrm{~d} x+\int_{\Omega \cap\{u \leq 1\}} u^{\beta+1} \mathrm{~d} x \\
& \leq \int_{\Omega}\left(u^{p}+u^{\beta+1}\right) \mathrm{d} x \leq \frac{1}{\Lambda_{1}} \int_{\Omega}|\nabla u|^{p} \mathrm{~d} x+\int_{\Omega} u^{\beta+1} \mathrm{~d} x
\end{aligned}
$$

where we used the Poincaré's inequality $\Lambda_{1}\|u\|_{L^{p}(\Omega)}^{p} \leq\|\nabla u\|_{L^{p}(\Omega)}^{p}$. Combining (4.4) with (4.5), we find that for

$$
D= \begin{cases}1-\alpha \max \left\{\frac{1}{\Lambda_{1}}, 1\right\}, & \text { if } \beta+1<q<p, \\ 1-\alpha, & \text { if } \beta+1=q<p, \\ 1-\frac{\alpha}{\Lambda_{1}}, & \text { if } \beta+1<q=p,\end{cases}
$$

there holds

$$
\frac{1}{2} y^{\prime}(t)+D \int_{\Omega}\left(|\nabla u|^{p}+u^{\beta+1}\right) \mathrm{d} x \leq 0
$$


Our next goal is to obtain the following differential inequality from (4.7):

$$
y^{\prime}(t)+K y(t)^{\gamma} \leq 0, \text { with } K>0,0<\gamma<1 .
$$

Integrating (4.8) with $t$ :

$$
y(t) \leq\left(y^{1-\gamma}(0)-K(1-\gamma) t\right)^{\frac{1}{1-\gamma}}
$$

which implies

$$
y(t) \rightarrow 0 \text { as } t \rightarrow T^{*}:=\frac{y^{1-\gamma}(0)}{K(1-\gamma)} .
$$

Thus, the finite time extinction for the solution of (1.1) is proved.

To obtain (4.8), we divided our proof into two parts: $p>\frac{2 N}{N+2}$ and $1<p<\frac{2 N}{N+2}$.

(i). If $p \geq \frac{2 N}{N+2}$, then $\frac{N p}{N-p} \geq 2$ for $p<N$ which implies that we can choose $r=2$ in (4.1). While if $p \geq N$, then $r \in[\beta+1,+\infty)$ which enables us to set $r=2$ in (4.1). In both cases, we can obtain

$$
\begin{aligned}
\|u\|_{L^{2}(\Omega)} & \leq C\|\nabla u\|_{L^{p}(\Omega)}^{\theta}\|u\|_{L^{\beta+1}(\Omega)}^{1-\theta}=C\left(\int_{\Omega}|\nabla u|^{p} \mathrm{~d} x\right)^{\frac{\theta}{p}}\left(\int_{\Omega} u^{\beta+1} \mathrm{~d} x\right)^{\frac{1-\theta}{\beta+1}} \\
& \leq C\left(\int_{\Omega}\left(|\nabla u|^{p}+u^{\beta+1}\right) \mathrm{d} x\right)^{\frac{\theta}{p}+\frac{1-\theta}{\beta+1}}
\end{aligned}
$$

from (4.1) with $r=2$. Then

$$
C^{-2} y(t) \leq\left(\int_{\Omega}\left(|\nabla u|^{p}+u^{\beta+1}\right) \mathrm{d} x\right)^{2\left(\frac{\theta}{p}+\frac{1-\theta}{\beta+1}\right)} .
$$

Combining (4.12) with (4.7), we can obtain (4.8) with

$$
\frac{1}{\gamma}=2\left(\frac{\theta}{p}+\frac{1-\theta}{\beta+1}\right)>1, K=2 D C^{-2 \gamma} .
$$

(ii). If $1<p<\frac{2 N}{N+2}$, let $2>r \in\left(\beta+1, \frac{N p}{N-p}\right]$ and $M=\|u\|_{L^{\infty}\left(Q_{T}\right)}$. Then we have

$$
y(t)=\|u\|_{L^{2}(\Omega)}=\int_{\Omega} u^{2-r} u^{r} \mathrm{~d} x \leq M^{2-r}\|u\|_{L^{r}(\Omega)}^{r} .
$$

By (4.1) with $r \in(\beta+1,2)$, there holds

$$
\begin{aligned}
y(t) & \leq M^{2-r}\left(C\|\nabla u\|_{L^{p}(\Omega)}^{\theta}\|u\|_{L^{\beta+1}(\Omega)}^{1-\theta}\right)^{r} \\
& \leq M^{2-r}\left(D^{\frac{\theta}{p}+\frac{1-\theta}{\beta+1}}\right)^{-r} C^{r}\left(D \int_{\Omega}\left(|\nabla u|^{p}+u^{\beta+1}\right) \mathrm{d} x\right)^{r\left(\frac{\theta}{p}+\frac{1-\theta}{\beta+1}\right)} .
\end{aligned}
$$

Combining (4.15) with (4.7), we can derive (4.8) with

$$
\frac{1}{\gamma}=r\left(\frac{\theta}{p}+\frac{1-\theta}{\beta+1}\right)>1, K=2 D M^{\gamma(r-2)} C^{-r \gamma} .
$$


Remark 4.2. In the case $1<p<2$, Fang, Wang and Li [8] obtained some similar extinction results. The results there needed stronger conditions for the coefficients of absorption and source terms. Moreover, the initial data was also been chosen small enough. However, our results hold for any nontrivial initial data and some $\alpha$ which needn't to be sufficiently small. Besides, our proof is also simpler.

Different from Theorem 4.1, the following theorem shows that finite time extinction can also occur for $q>p$ and $1<p<2$ with small initial data.

Theorem 4.2. Assume that $q>p, 1<p<2$, then the solution of (1.1) will vanish at finite time provided the initial data is small enough.

Proof. The proof here is same as the one in [33, Theorem 4.1], we omit it.

4.2. Decay. Let us now consider the decay of the solution.

Theorem 4.3. Assume that $\beta \geq 1$ and $p \geq 2$, then the solution of (1.1) will not extinguish in finite time. Assume additionally $\beta \leq q-1$, then there exists a constant $\epsilon>0$, such that if $u_{0} \geq 0$ and $\left\|u_{0}\right\|_{L^{\infty}(\Omega)}<\epsilon$, then the solution will decay to zero as $t \rightarrow+\infty$. Moreover, we have the following estimates:

$$
\left\{\begin{array}{l}
0 \leq u \leq C_{1}\left(t+C_{2}\right)^{-\gamma}, \gamma=\frac{1}{\beta-1}, \text { for } 1<\beta \leq q-1 \\
0 \leq u \leq C_{3} \mathrm{e}^{-C_{4} t}, \text { for } \beta=1, q \geq 2 .
\end{array}\right.
$$

The constants $C_{i}, i=1,2,3,4$ appeared above depend on $q, \beta, \alpha$.

Proof. By [15, Theorem 3.3], we know that the solution of

$$
\left\{\begin{aligned}
v_{t}-\Delta_{p} v & =-|v|^{\beta-1} v, & x \in \Omega, t>0, \\
v & =0, & x \in \partial \Omega, t>0, \\
v(x, 0) & =u_{0}(x), & x \in \Omega .
\end{aligned}\right.
$$

will not extinguish in finite time if $p \geq 2, \beta \geq 1, u_{0}(x) \in W_{0}^{1, p}(\Omega) \cap L^{\infty}(\Omega), u_{0}(x) \not \equiv 0$. As was shown in Theorem 3.1, $u \geq 0$. Thus, $v$ is a sub-solution of (1.1). By the comparison principle, $u$ will not extinguish in finite time.

Let us now consider the decay of the solution of (1.1). For convenience, we define $\mathscr{L}_{p}$ as: $\mathscr{L}_{p} \varphi=\varphi_{t}-\Delta_{p} \varphi-\alpha|\varphi|^{q-2} \varphi+|\varphi|^{\beta-1} \varphi$.

If $1<\beta \leq q-1$, let

$$
w(x, t)=C_{1}\left(t+C_{2}\right)^{-\gamma}, \gamma=\frac{1}{\beta-1},
$$

where $C_{1}, C_{2}>0$ are constants to be decided later. By a direct computation, we have

$$
\mathscr{L}_{p} w=\left(t+C_{2}\right)^{-\gamma-1}\left(-\gamma C_{1}+C_{1}^{\beta}-\alpha C_{1}^{q-1}\left(t+C_{2}\right)^{-(q-1-\beta) \gamma}\right) .
$$

If $\beta<q-1$, let $C_{1}, C_{2}>0$ satisfy: $(2 \gamma)^{\gamma} \leq C_{1} \leq(2 \alpha)^{\frac{1}{\beta-q+1}} C_{2}^{\gamma}$, then we have $\mathscr{L}_{p} w \geq$ 0 . Assume additionally that $\left\|u_{0}\right\|_{L^{\infty}(\Omega)} \leq C_{1} C_{2}^{-\gamma}$, then we have $w(x, 0) \geq u_{0}(x)$. 
Thus, we have shown that $C_{1}, C_{2}$ satisfy

$$
\max \left\{\left\|u_{0}\right\|_{L^{\infty}(\Omega)} C_{2}^{\gamma},(2 \gamma)^{\gamma}\right\} \leq(2 \alpha)^{\frac{1}{\beta-q+1}} C_{2}^{\gamma} .
$$

In order (4.21) to be satisfied, we need

$$
\left\|u_{0}\right\|_{L^{\infty}(\Omega)} \leq \epsilon:=(2 \alpha)^{\frac{1}{\beta-q+1}}
$$

and

$$
C_{2} \geq 2^{\frac{1}{\gamma(q-\beta-1)}+1} \gamma
$$

For $C_{1}, C_{2}$ satisfying (4.21) and (4.23), we know that $w$ is a super-solution, which implies that

$$
0 \leq u \leq C_{1}\left(t+C_{2}\right)^{-\gamma}, \gamma=\frac{1}{\beta-1}, \text { for } 1<\beta<q-1
$$

provided $u_{0}$ satisfies (4.22).

If $1<\beta=q-1$, assume additionally that $\alpha<1$, we can still obtain the first estimate in (4.17) for $C_{1}, C_{2}$ and $u_{0}$ satisfying

$$
C_{1} \geq \max \left\{C_{2}^{\gamma},\left(\frac{\gamma}{1-\alpha}\right)^{\gamma}\right\} .
$$

If $\beta=1, q \geq 2$, let

$$
w=C_{1} \mathrm{e}^{-C_{2} t}
$$

with

$$
\left\{\begin{array}{l}
\alpha C_{1}^{q-2}+C_{2} \leq 1,\left\|u_{0}\right\|_{L^{\infty}(\Omega)} \leq C_{1}, \text { for } q>2 \\
0<C_{2} \leq 1-\alpha,\left\|u_{0}\right\|_{L^{\infty}(\Omega)} \leq C_{1}, \text { for } q=2 .
\end{array}\right.
$$

We can still verify that $w$ is a super-solution of (1.1). Then we obtain the desired result by comparison principle. Thus, the proof is complete.

\section{Finite time Blowup}

In this section we will use two different methods to show that the solution of (1.1) will blow up in finite time. We first introduce the following blowup result which is based on the construction of a self-similar sub-solution and the comparison principle.

Theorem 5.1. Suppose that $q>\max \{p, 2, \beta+1\}$. Then the solution of (1.1) will blow up in finite time for some large $u_{0}(x)$ satisfying $u_{0}(x)>0$ in $\Omega^{\prime} \subset \Omega$.

Proof. Without loss of generality, we assume that $0 \in \Omega$. Define $v(x, t)$ as:

$$
v(x, t)=\frac{1}{(1-\varepsilon t)^{k}} V\left(\frac{|x|}{(1-\varepsilon t)^{m}}\right), t_{0} \leq t<\frac{1}{\varepsilon},
$$

where

$$
V(y)=1+\frac{A}{\sigma}-\frac{y^{\sigma}}{\sigma A^{\sigma-1}}, y \geq 0,
$$


and

$$
\sigma=\frac{p}{p-1}, k=\frac{1}{q-2}, 1<m<\frac{q-p}{p(q-2)}, A>\frac{2 k}{m}, 0<\varepsilon<\frac{\alpha}{k\left(1+\frac{A}{\sigma}\right)}
$$

Let

$$
R=\left(A^{\sigma-1}(\sigma+A)\right)^{\frac{1}{\sigma}}, D:=\left\{(x, t)\left|t_{0} \leq t<\frac{1}{\varepsilon},\right| x \mid<R(1-\varepsilon t)^{m}\right\}
$$

then $V(y) \geq 0$ is smooth in $D$ and $v(y)<0$ if $y>R$. Moreover, $V(y)$ satisfies

$$
\begin{cases}1 \leq V(y) \leq 1+\frac{A}{\sigma},-1 \leq V^{\prime}(y) \leq 0, & \text { if } 0 \leq y \leq A \\ 0 \leq V(y) \leq 1,-\frac{R^{\sigma-1}}{A^{\sigma-1}} \leq V^{\prime}(y) \leq-1, & \text { if } A \leq y \leq R \\ \left(\left|V^{\prime}\right|^{p-2} V^{\prime}\right)^{\prime}+\frac{N-1}{y}\left|V^{\prime}\right|^{p-2} V^{\prime}=-\frac{N}{A} . & \end{cases}
$$

Define

$$
\mathscr{L}_{p} v=v_{t}-\Delta_{p} v-\alpha|v|^{q-2} v+|v|^{\beta-1} v
$$

then

$$
\mathscr{L}_{p} v=\frac{\varepsilon\left(k V+m y V^{\prime}\right)}{(1-\varepsilon t)^{k+1}}-\frac{\left(\left|V^{\prime}\right| p^{p-2} V^{\prime}\right)^{\prime}+\frac{N-1}{y}\left|V^{\prime}\right|^{p-2} V^{\prime}}{(1-\varepsilon t)^{(k+m)(p-1)+m}}-\frac{\alpha V^{q-1}}{(1-\varepsilon t)^{k(q-1)}}+\frac{V^{\beta}}{(1-\varepsilon t)^{k \beta}} .
$$

By (5.3), we can easily see that $k+1=k(q-1), k \beta<k+1,(k+m)(p-1)+m<k+1$. Then, for $0 \leq \frac{1}{\varepsilon}-t_{0} \ll 1$ and $t_{0} \leq t<\frac{1}{\varepsilon}$, if $y \in[0, A]$,

$$
\begin{aligned}
\mathscr{L}_{p} v= & \frac{1}{(1-\varepsilon t)^{k+1}}\left\{\varepsilon\left(k V+m y V^{\prime}\right)+\frac{N}{A}(1-\varepsilon t)^{k+1-m-(k+m)(p-1)}-\alpha V^{q-1}\right. \\
& \left.+V^{\beta}(1-\varepsilon t)^{k+1-k \beta}\right\} \\
\leq & \frac{1}{(1-\varepsilon t)^{k+1}}\left\{\varepsilon k\left(1+\frac{A}{\sigma}\right)+\frac{N}{A}(1-\varepsilon t)^{k+1-m-(k+m)(p-1)}-\alpha\right. \\
& \left.+V^{\beta}(1-\varepsilon t)^{k+1-k \beta}\right\} \\
\leq & 0, \text { for } \varepsilon \ll \frac{\alpha}{k\left(1+\frac{A}{\sigma}\right)} .
\end{aligned}
$$


Similarly, if $y \in[A, R]$,

$$
\begin{aligned}
\mathscr{L}_{p} v & \leq \frac{1}{(1-\varepsilon t)^{k+1}}\left\{\varepsilon(k-m A)+\frac{N}{A}(1-\varepsilon t)^{k+1-m-(k+m)(p-1)}+(1-\varepsilon t)^{k+1-k \beta}\right\} \\
& \leq 0 .
\end{aligned}
$$

Thus, we have prove that $\mathscr{L}_{p} v \leq 0$ in $D$. In order for $v(x, t)$ to be a sub-solution, we also need to choose suitable initial data and boundary value. Let $t_{0}$ be such that $u_{0}(x)>0$ in $B\left(0, R\left(1-\varepsilon t_{0}\right)^{m}\right) \subset \Omega$ and $u_{0}(x) \geq v\left(\cdot, t_{0}\right)$ in $B\left(0, R\left(1-\varepsilon t_{0}\right)^{m}\right)$. According to Theorem 3.1 and the definition of $v, u(x, t) \geq 0=v(x, t)$ in $\partial B(0, R(1-$ $\left.\varepsilon t)^{m}\right) \times\left(t_{0}, \frac{1}{\varepsilon}\right)$. Thus, we have shown that $v\left(x, t+t_{0}\right)$ is a sub-solution for (1.1) in $D\left(t_{0}\right):=\left\{(x, t)\left|0 \leq t \leq \frac{1}{\varepsilon}-t_{0},\right| x \mid<R\left(1-\varepsilon\left(t+t_{0}\right)\right)^{m}\right\}$. By Proposition 2.1,

$$
u(x, t) \geq v\left(x, t+t_{0}\right),(x, t) \in D\left(t_{0}\right) .
$$

Noticing that $\lim _{t \rightarrow 1 / \varepsilon} v(0, t) \rightarrow+\infty$, we have $u$ must blow up at a finite time $T \leq$ $\frac{1}{\varepsilon}-t_{0}<\infty$.

Remark 5.1. If $1<p<2$ we can also choose $m$ such that $0<m<\frac{2-p}{p(q-2)}$ in (5.3).

Remark 5.2. The method we used above is first introduced by Souplet and Weissler in [28] for $p=2$. Li and Xie developed this method in [20] for $p>2$. In our latest papers [34, 35], we used this method to study the blowup results of the initial boundary problem for a p-Laplacian parabolic equation with a nonlinear gradient term.

Next, we will introduce some blowup results whose proofs are based on the energy method and concavity method which were also used in [1, 20, 33, 36] and the references therein. In the proof of our desired results, the following lemma concerning the socalled "energy" is useful.

Lemma 5.1. Let

$$
E(t)=\int_{\Omega}\left(\frac{1}{p}|\nabla u|^{p}+\frac{1}{\beta+1} u^{\beta+1}-\frac{\alpha}{q} u^{q}\right) \mathrm{d} x .
$$

If $E(0)<0$, then $E(t)<0$ for all $t>0$.

Proof. By a direct computation, we can see that

$$
\begin{aligned}
E^{\prime}(t) & =\int_{\Omega}\left(|\nabla u|^{p-2} \nabla u \cdot \nabla u_{t}+u^{\beta} u_{t}-\alpha u^{q-1} u_{t}\right) \mathrm{d} x \\
& =\int_{\Omega}\left(-\Delta_{p} u+u^{\beta}-\alpha u^{q-1}\right) u_{t} \mathrm{~d} x=-\int_{\Omega} u_{t}^{2} \mathrm{~d} x \leq 0 .
\end{aligned}
$$

Hence, $E(t) \leq E(0)<0$ for all $t>0$.

The following theorem is the main result of this section. 
Theorem 5.2. Suppose $u_{0}(x)$ satisfies

$$
\int_{\Omega}\left(\frac{1}{p}\left|\nabla u_{0}\right|^{p}+\frac{1}{\beta+1} u_{0}^{\beta+1}-\frac{\alpha}{q} u_{0}^{q}\right) \mathrm{d} x<0 .
$$

then the solution of (1.1) will blow up in finite time provided that one of the following cases occurs:

(a) $0<\beta<\min \{1, p-1\}, q>\max \{p, 2\}$;

(b) $q=p, 1<\beta<p-1$;

(c) $\beta=p-1, q>\max \{p, 2\}$;

(d) $1<\beta<p-1, q>p>2$;

(e) $\beta+1=q=p>2$.

(f) $q>\beta+1>p>2$, and $\left\|u_{0}\right\|_{L^{2}(\Omega)}^{2}$ is large enough.

Proof. Let $y(t)=\|u\|_{L^{2}(\Omega)}^{2}$, then it satisfies

$\frac{1}{2} y^{\prime}(t)=\int_{\Omega} u u_{t} \mathrm{~d} x=\int_{\Omega}\left(u \Delta_{p} u-u^{\beta+1}+\alpha u^{q}\right) \mathrm{d} x=\int_{\Omega}\left(-|\nabla u|^{p}-u^{\beta+1}+\alpha u^{q}\right) \mathrm{d} x$.

By Lemma 5.1, we can get

$$
\begin{aligned}
\frac{1}{2 p} y^{\prime}(t) & =\int_{\Omega}\left(-\frac{1}{p}|\nabla u|^{p}-\frac{1}{p} u^{\beta+1}-\frac{\alpha}{p} u^{q}\right) \mathrm{d} x \\
& =-E(t)+\left(\frac{1}{\beta+1}-\frac{1}{p}\right) \int_{\Omega} u^{\beta+1} \mathrm{~d} x+\alpha\left(\frac{1}{p}-\frac{1}{q}\right) \int_{\Omega} u^{q} \mathrm{~d} x \\
& >\left(\frac{1}{\beta+1}-\frac{1}{p}\right) \int_{\Omega} u^{\beta+1} \mathrm{~d} x+\alpha\left(\frac{1}{p}-\frac{1}{q}\right) \int_{\Omega} u^{q} \mathrm{~d} x
\end{aligned}
$$

Let us now estimate (5.15) furthermore in different cases.

(a). $0<\beta<\min \{1, p-1\}, q>\max \{p, 2\}$. In this case, by Hölder's inequality, (5.15) can be rewritten as

$$
\frac{1}{2 p} y^{\prime}(t) \geq \alpha\left(\frac{1}{p}-\frac{1}{q}\right) \int_{\Omega} u^{q} \mathrm{~d} x \geq \alpha\left(\frac{1}{p}-\frac{1}{q}\right)|\Omega|^{\frac{2-q}{2}} y^{\frac{q}{2}},
$$

i.e.

$$
y^{\prime}(t) \geq 2 p \alpha\left(\frac{1}{p}-\frac{1}{q}\right)|\Omega|^{\frac{2-q}{2}} y^{\frac{q}{2}}(t) .
$$

Integrating (5.17) in $t$, we have

$$
y(t) \geq\left(y^{\frac{2-q}{2}}(0)-p \alpha(q-2)\left(\frac{1}{p}-\frac{1}{p}\right)|\Omega|^{\frac{2-q}{2}} t\right)^{\frac{2}{2-q}}
$$

which implies that

$$
y(t) \rightarrow+\infty, \text { as } t \rightarrow T_{1}^{*}:=\frac{y^{\frac{2-q}{2}}(0)|\Omega|^{\frac{2}{2-q}}}{p \alpha(q-2)\left(\frac{1}{p}-\frac{1}{q}\right)} .
$$


(b). $q=p, 1<\beta<p-1$. In this case, there holds

$$
y^{\prime}(t)>2 p\left(\frac{1}{\beta+1}-\frac{1}{p}\right) \int_{\Omega} u^{\beta+1} \mathrm{~d} x \geq 2 p\left(\frac{1}{\beta+1}-\frac{1}{p}\right)|\Omega|^{\frac{1-\beta}{2}} y^{\frac{\beta+1}{2}}(t) .
$$

Then

$$
y(t) \geq\left(y^{\frac{1-\beta}{2}}(0)-p(\beta-1)\left(\frac{1}{\beta+1}-\frac{1}{p}\right)|\Omega|^{\frac{1-\beta}{2}} t\right)^{\frac{2}{1-\beta}} .
$$

Thus

$$
y(t) \rightarrow+\infty, \text { as } t \rightarrow T_{2}^{*}:=\frac{y^{\frac{1-\beta}{2}}(0)|\Omega|^{\frac{2}{1-\beta}}}{p(\beta-1)\left(\frac{1}{\beta+1}-\frac{1}{p}\right)} .
$$

(c). $\beta=p-1, q>\max \{p, 2\}$. Similarly as (1), we can derive that $y(t) \rightarrow+\infty$, as $t \rightarrow T_{3}^{*}=T_{1}^{*}$.

(d). $1<\beta<p-1, q>p>2$. We can rewrite (5.15) as

$$
\begin{aligned}
y^{\prime}(t) & \geq 2 p\left(\frac{1}{\beta+1}-\frac{1}{p}\right)|\Omega|^{\frac{1-\beta}{2}} y^{\frac{\beta+1}{2}}(t)+2 p \alpha\left(\frac{1}{p}-\frac{1}{q}\right)|\Omega|^{\frac{2-q}{2}} y^{\frac{q}{2}}(t) \\
& \geq 4 p \sqrt{\alpha\left(\frac{1}{\beta+1}-\frac{1}{p}\right)\left(\frac{1}{p}-\frac{1}{q}\right)}|\Omega|^{\frac{3-q-\beta}{4}} y^{\frac{q+\beta+1}{4}}(t) .
\end{aligned}
$$

Then $y(t) \rightarrow+\infty$, as $t \rightarrow T_{4}^{*} \leq \min \left\{T_{1}^{*}, T_{2}^{*}, T^{\prime}\right\}$ with

$$
T^{\prime}:=\frac{y^{\frac{3-q-\beta}{4}}(0)|\Omega|^{\frac{4}{3-q-\beta}}}{p(q+\beta-3) \sqrt{\alpha\left(\frac{1}{\beta+1}-\frac{1}{p}\right)\left(\frac{1}{p}-\frac{1}{q}\right)}} .
$$

(e). $\beta+1=q=p$. If this happens, then we can only derive from (5.15) that $y^{\prime}(t)>0$ which can not be used to show that $y(t) \rightarrow+\infty$ as $t \rightarrow \widetilde{T}<\infty$. However, if $p>2$, we can still obtain desired result by the concavity method. The proof here is same as the one of [20, Lemma 3.4], here we just provided the final ordinary inequality below:

$$
y^{\prime}(t) \geq \frac{y^{\prime}(0)}{y^{\frac{p}{2}}(0)} y^{\frac{p}{2}}(t) .
$$

(f). $q>\beta+1>p>2$. As $\beta+1>p$, the first term of the right side hand in (5.15) is negative, we cannot use the procedure above directly. However, by the fact that $q>\beta+1$, we can still obtain the desired result. Indeed, by Young's inequality, we have for small $\epsilon>0$

$$
\int_{\Omega} u^{\beta+1} \mathrm{~d} x \leq \frac{\epsilon(\beta+1)}{q} \int_{\Omega} u^{q} \mathrm{~d} x+C(\epsilon) \frac{q-\beta-1}{q}|\Omega| .
$$

Choose a suitable $\epsilon$ such that

$$
\left(\frac{1}{\beta+1}-\frac{1}{p}\right) \frac{\epsilon(\beta+1)}{q} \geq-\frac{\alpha}{2}\left(\frac{1}{p}-\frac{1}{q}\right) .
$$


Then we have

$$
\frac{1}{2 p} y^{\prime}(t) \geq \frac{\alpha}{2}\left(\frac{1}{p}-\frac{1}{q}\right) \int_{\Omega} u^{q} \mathrm{~d} x+C(\epsilon)\left(\frac{1}{\beta+1}-\frac{1}{p}\right) \frac{q-\beta-1}{q}|\Omega| .
$$

If we assume additionally that $\left\|u_{0}\right\|_{L^{2}(\Omega)}^{2}$ is large enough, then we can derive

$$
y^{\prime}(t) \geq \frac{p \alpha}{2}\left(\frac{1}{p}-\frac{1}{q}\right)|\Omega|^{\frac{2-q}{2}} y^{\frac{q}{2}}(t)
$$

which implies that

$$
y(t) \rightarrow+\infty, \text { as } t \rightarrow T_{5}^{*}:=4 T_{1}^{*} .
$$

The proof of Theorem 5.2 is now complete.

Remark 5.3. Following the same manner as in [20, Theorem 3.5], we can still obtain the desired blowup results in case (e) of Theorem 5.2 if we assume that $\alpha>\Lambda_{1}+1$ instead of (5.13).

Remark 5.4. During the proof of Theorem 5.2, we also obtain an upper bound of the blowup time in each case.

\section{Discussions}

As was shown in the previous sections, the relation of $p, q, \beta$ plays an important role in determining the properties of the weak solution of (1.1). To be specific, we will state it for $1<p<2$ and $p>2$ respectively. Moreover, we will use two figures to state the results of blowup, extinction and global existence intuitionally. For simplicity, we will not point out which domain the boundary lines and the coordinate axis belong to.

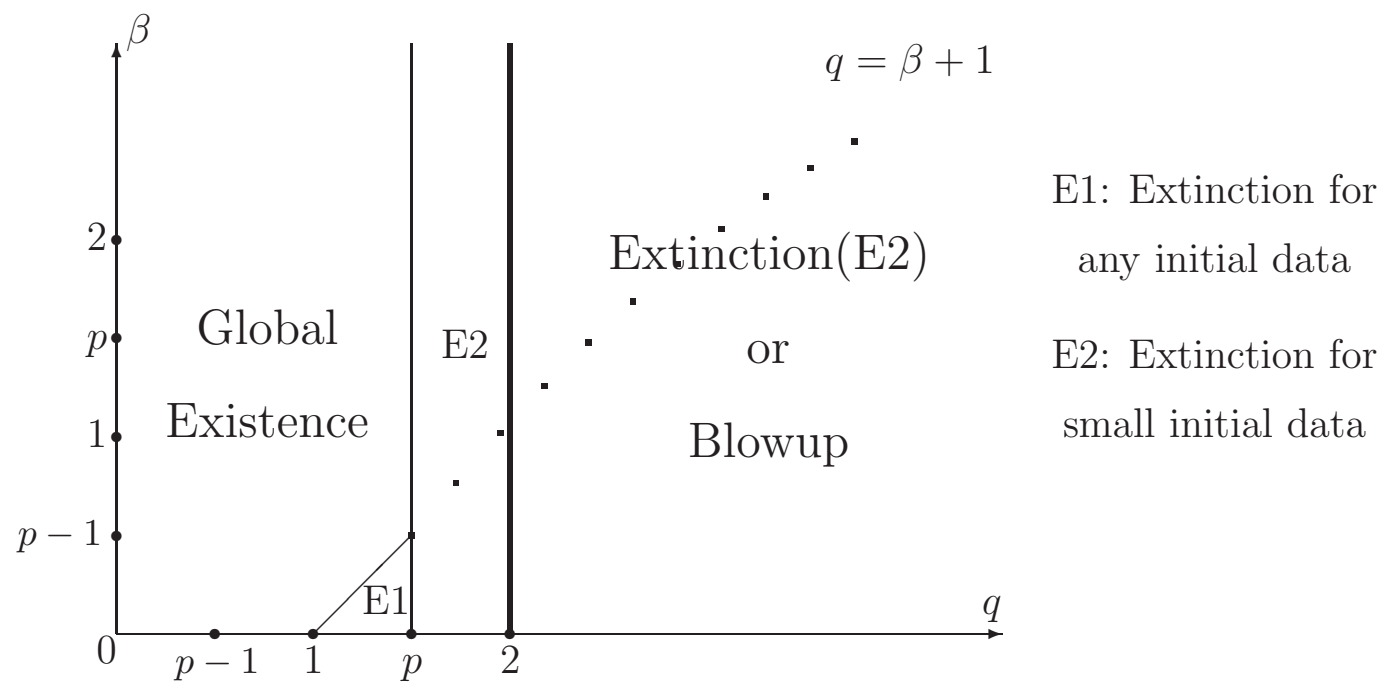

FiguRE 1. $1<p<2$ 
We first discuss the case $1<p<2$ (Figure 1). In this case, if $q>\max \{2, \beta+1\}$ or $0<\beta \leq p-1, q>2$, then finite time blowup will occur for some suitably large initial data, see Theorem 5.1 and 5.2((a),(c)). If $q \in(\beta+1, p)$, or $q=\beta+1$, or $q=p$, then finite time extinction will happen with suitable $\alpha$ and any nontrivial initial data, see Theorem 4.1. While if $q>p, \beta>0$, then small initial data can lead to finite time extinction, see Theorem 4.2. Noticing that if $q>2$, then large initial data can lead to finite time blowup while small initial data implies finite time extinction which is interesting.

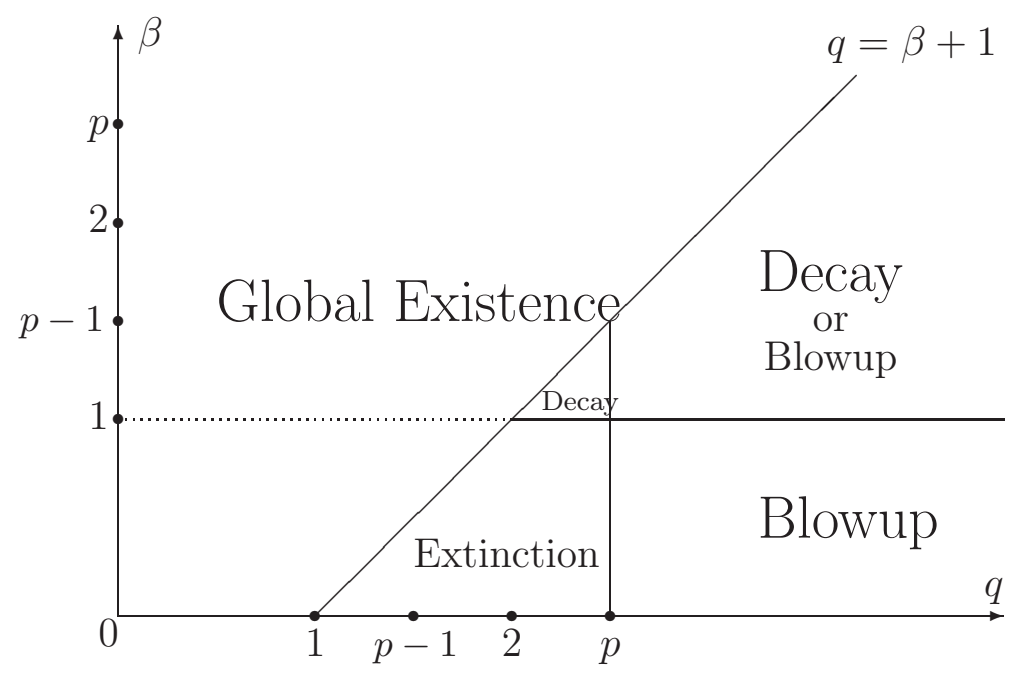

FiguRE 2. $p>2$

Next, let us consider the case $p>2$ (Figure 2). In this case, if $q>\max \{p, \beta+1\}$, or $q>p, 0<\beta \leq q-1$, or $q=p \geq \beta+1>2$, then for some suitably large initial data, the solution of (1.1) will blow up in finite time, see Theorem 5.1 and Theorem 5.2((b),(d),(e),(f)). If $q \in(\beta+1, p), \beta<1$, or $q=\beta+1<2$, or $q=p$, then finite time extinction will happen with suitable $\alpha$ and any nontrivial initial data, see Theorem 4.1. Besides, if $1 \leq \beta \leq q-1$, then as was shown in Theorem 4.3, the solution of (1.1) cannot extinction in finite time, while it will decay to zero as $t \rightarrow+\infty$ for some suitably small $u_{0}$.

We also need to point out that finite time extinction is not a singularity property for solution of (1.1) as $\beta$ and $q-1$ are positive. If finite time extinction happens, we have in fact shown that the solution of (1.1) is global in time bounded which is also an important property of the solution of (1.1). For the global existence of the weak solution, we can see from Theorem 3.2 that the critical value for $q$ is $p$ if $1<p<2$. While in the degenerate case, the critical value is $p$ and $\beta+1$. Moreover, if $q \leq p$ or $q<\beta+1$, then we can obtain the global existence. 


\section{ACKNOWLEDGEMENT}

This work was supported in part by the National Natural Science Foundation of China (No. 11371286, 11401458), the Special Fund of Education Department (No. 2013JK0586) and the Youth Natural Science Grant (No. 2013JQ1015) of Shaanxi Province of China.

\section{REFERENCES}

[1] S. Antontsev, J. I. Díaz and S. Shmarev, Energy methods for free boundary problems. Applications to nonlinear PDEs and Fluid Mechanics, Series Progress in Nonlinear Dierential Equations and Their Applications, No. 48, Birkäuser, Boston, 2002.

[2] S. Antontsev and S. Shmarev, Anisotropic parabolic equations with variable nonlinearity, Publ. Mat., 53 (2009), pp. 355-399.

[3] S. Antontsev and S. Shmarev, Energy solutions of evolution equations with nonstandard growth conditions, Monografías de la Real Academia de Ciencias de Zargoza, 38 (2012), pp. 85-111.

[4] A. Attouchi, Well-posedness and gradient blow-up estimate near the boundary for a HamiltonJacobi equation with degenerate diffusion, J. Differential Equations, 253 (2012), pp. 2474-2492.

[5] J. Dávila and M. Montenegro, Existence and asymptotic behavior for a singular parabolic equation, Trans. Amer. Math. Soc., 357 (2005), pp. 1801-1828.

[6] J. I. Díaz, Qualitative study of nonlinear parabolic equations: an introduction, Extracta Math., 16(3) (2001), pp. 303-341.

[7] E. DiBenedetto, Degenerate Parabolic Equations, Springer-Verlag, New York, 1993.

[8] Z. B. Fang, M. Wang and G. Li, Extinction properties of solutions for a p-Laplacian evolution equation with nonlinear source and strong absorption, Mathematica Aeterna, 3 (2013), pp. 579-591.

[9] Z. B. Fang, G. Li, Extinction and decay estimates of solutions for a class of doubly degenerate equations, Appl. Math. Lett., 25 (2012), pp. 1795-1802.

[10] Z. B. Fang, X. H. Xu, Extinction behavior of solutions for the p-Laplacian equations with nonlocal sources, Nonlinear Anal. Real World Appl. 13 (2012), pp. 1780-1789.

[11] H. Fujita, On the blowing up of solutions of the Cauchy problem for $u_{t}=\Delta u+u^{1+\alpha}$, J. Fac. Sci. Univ. Tokyo Sect. A. Math., 16 (1966), pp. 105-113.

[12] V. A. Galaktionov and J. L. Vázquez, Continuation of blowup solutions of nonlinear heat equations in several space dimensions, Comnm. Pure Appl. Math., 50 (1997), pp. 1-67.

[13] V. A. Galaktionov and S. A. Posashkov, Single point blow-up for N-dimensional qusilinear equations with gradient diffusion and source, Indiana Univ. Math. J., 40 (1991), pp. 1041-1060.

[14] J. Giacomoni, P. Sauvy and S. Shmarev, Complete quenching for a quasilinear parabolic equation, J. Math. Anal. Appl. 410 (2014), pp. 607-624.

[15] Y. G. Gu, Necessary and sufficient conditions of extinction of solution on parabolic equations, Acta. Math. Sin. 37 (1994), pp. 73-79.

[16] M. Hesaaraki and A. Moameni, Blow-up of positive solutions for a family of nonlinear parabolic equations in general domain in $\mathbb{R}^{N}$, Michigan Math. J., 52 (2004), pp. 375-389.

[17] C. H. Jin, J. X. Yin and S. N. Zheng, Critical Fujita absorption exponent for evolution pLaplacian with inner absorption and boundary flux, Diff. Integral Equations, 27 (2014), pp. 643-658.

[18] Y. C. Kwong, Boundary behavior of the fast diffusion equation, Trans. Amer. Math. Soc., 322 (1990), pp. 263-283.

[19] H. A. Levine and L. E. Payne, Nonexistence of global weak solutions for classes of nonlinear wave and parabolic equations, J. Math. Anal. Appl., 55 (1976), pp.329-334.

[20] Y. X. Li and C. H. Xie, Blow-up for p-Laplacian parabolic equations, E. J. Differential Equations, 2003(20) (2003), pp. 1-12. 
[21] P. Lindqvist, Notes on the p-Laplace equation, http://www.math.ntnu.no/ lqvist/p-laplace.pdf, 2006.

[22] I. Ly, The first eigenvalue for the p-Laplacian operator, J. Inequal. Pure Appl. Math., 6 (2005), Article 91, 12 pp.

[23] C. L. Mu and R. Zeng, Single-point blow-up for a doubly degenerate parabolic equation with nonlinear source, Proceedings of the Royal Society of Edinburgh, 141A (2011), pp. 641-654.

[24] C. Y. Qu, X. L. Bai and S. N. Zheng, Blow-up versus extinction in a nonlocal p-Laplace equation with Neumann boundary conditions, J. Math. Anal. Appl., 412 (2014), pp. 326-333.

[25] P. Quittner, Blow-up for semilinear parabolic equations with a gradient term, Math. Methods Appl. Sci., 14 (1991), 413-417.

[26] P. Quittner and Ph. Souplet, Superlinear Parabolic Problems: Blow-up, Global Existence and Steady States, Birkhäuser, 2007.

[27] J. Simon, Compact sets in the space $L^{p}(0, T ; B)$, Ann. Mat. Pura Appl., 146 (1987), pp. 65-96.

[28] Ph. Souplet and F. B. Weissler, Self-similar subsolutions and blowup for nonlinear parabolic equations, J. Math. Anal. Appl., 212 (1997), pp. 60-74.

[29] J. L. Vázquez, Smoothing and decay estimates for nonlinear diffusion equations: equations of porous medium type, Oxford Univ. Press, Oxford, 2006.

[30] C. P. Wang, S. N. Zheng and Z. J. Wang, Critical Fujita exponents for a class of quasilinear equations with homogeneous Neumann boundary data, Nonlinearity, 20 (2007), pp. 1343-1359.

[31] M. Winkler, A strongly degenerate diffusion equation with strong absorption, Math. Nachr., 227 (2004), pp. 83-101.

[32] J. G. Yang, C. X. Yang and S. N. Zheng, Second critical exponent for evolution p-Laplacian equation with weighted source, Math. Comput. Modelling, 56 (2012), pp. 247-256.

[33] J. X. Yin and C. H. Jin, Critical extinction and blow-up exponents for fast diffusive p-Laplacian with sources, Math. Methods Appl. Sci., 30 (2007), pp. 1147-1167.

[34] Z. C. Zhang and Y. Li, Blowup and existence of global solutions to nonlinear parabolic equations with degenerate diffusion, E. J. Differential Equations, 2013 (264) (2013), pp. 1-17.

[35] Z. C. Zhang and Y. Li, Classification of blowup solutions for a parabolic p-Laplacian equation with nonlinear gradient terms, J. Math. Anal. Appl., 436 (2016), pp. 1266-1283.

[36] J. N. Zhao, Existence and nonexistence of solutions for $u_{t}=\operatorname{div}\left(|\nabla u|^{p-2} \nabla u\right)+f(\nabla u, u, x, t)$, J. Math. Anal. Appl., 172 (1993), pp. 130-146.

[37] J. N. Zhao and Z. L. Liang, Blow-up rate of solutions for p-Laplacian equation, J. Partial Diff. Equ., 21 (2008), pp. 134-140.

[38] J. Zhou, Global existence and blow-up of solutions for a Non-Newton polytropic filtration system with special volumetric moisture content, Comput. Math. Appl., 71 (2016), pp. 1163-1172.

[39] J. Zhou and D. Yang, Upper bound estimate for the blow-up time of an evolution m-Laplace equation involving variable source and positive initial energy, Comput. Math. Appl., 69 (2015), pp. 1463-1469.

School of Mathematics and Statistics, Xi'an Jiaotong University, Xi'an, 710049, P. R. ChinA

E-mail address: zhangzc@mail.xjtu.edu.cn, liyan1989@stu.xjtu.edu.cn

College of Science, Xi'an University of Architecture \& Technology, Xi'an, 710055,

P. R. China

E-mail address: $78184385 @ q q . c o m$ 